\title{
Novel solitons in parametric amplifiers and atom lasers
}

\author{
P D Drummond $\dagger, \mathrm{K}$ V Kheruntsyan $\dagger$ and $\mathrm{H} \mathrm{He} \ddagger$ \\ $\dagger$ Department of Physics, University of Queensland, St Lucia, QLD 4072, Australia \\ $\ddagger$ Department of Theoretical Physics, School of Physics, A28, Sydney University, NSW \\ 2006, Australia
}

Received 11 January 1999

\begin{abstract}
We review recent developments in quantum and classical soliton theory, leading to the possibility of observing both classical and quantum parametric solitons in higher-dimensional environments. In particular, we consider the theory of three bosonic fields interacting via both parametric (cubic) and quartic couplings. In the case of photonic fields in a nonlinear optical medium this corresponds to the process of sum frequency generation (via $\chi^{(2)}$ nonlinearity) modified by the $\chi^{(3)}$ nonlinearity. Potential applications include an ultrafast photonic AND-gate. The simplest quantum solitons or energy eigenstates (bound-state solutions) of the interacting field Hamiltonian are obtained exactly in three space dimensions. They have a point-like structure-even though the corresponding classical theory is nonsingular. We show that the solutions can be regularized with the imposition of a momentum cut-off on the nonlinear couplings. The case of three-dimensional matter-wave solitons in coupled atomic/molecular Bose-Einstein condensates is discussed.
\end{abstract}

Keywords: Nonlinear optics, atom optics, classical parametric solitons, spatio-temporal, two and three dimensional, optical logic gates, quantum solitons, atomic and molecular Bose-Einstein condensates

\section{Introduction}

The classical view of solitons is that they are stable nonlinear waveforms, which are spatially localized, yet show neither dissipation nor dispersion. In some moving frame with time coordinate $\tau$, this implies that for a complex field $\phi_{j}$, the time-dependent solution must satisfy: $\phi_{j}(\tau)=\phi_{j}(0) \mathrm{e}^{\mathrm{i} \omega_{j} \tau}$. An early example is the nonlinear wave in shallow water [1], described by the KdV equation [2]. In applied mathematics, it is common to use an even more restrictive definition, with conditions on the collisions as well. In this case, the solutions we refer to as solitons, would be called 'solitary waves' or just 'lumps' [3]. A modern example is the short optical pulse in a single-mode fibre, which is rather precisely described by the nonlinear Schrödinger (NLS) equation [4], and is the subject of many recent experiments [5]. These, however, are restricted to one space dimension.

In the quantum domain, there must be corresponding objects, and one may even expect these to show more structure than their classical counterparts. One view [6] is that a quantum soliton is an energy eigenstate of a local quantum field theory, which has a spatially localized correlation function, so that: $\hat{H}|\Psi\rangle=E|\Psi\rangle$. It is known that, at least for sufficiently weak couplings, a quantum soliton exists for every classical soliton [6]. Early examples of this were various theoretical models in high-energy physics. However, until recently, the experimental evidence for quantum effects in solitons was extremely scarce. This situation has changed dramatically due to the prediction [7] and observation [8] of quantum effects in optical pulses in fibres, which can be regarded as photonic bound states.

Despite these developments, there are many unsolved problems in soliton theory. The following questions seem to be of particular significance:

- When can classical solitons form in more than one dimension?

- When can quantum solitons physically occur?

- Are there essential differences between quantum and classical solitons?

- Do solitons require integrable dynamical equations?

- Are there applications-for example, in communications or computing?

- Is there any novel physics to be found, related to different quantum statistics?

In this paper, we review some recent developments which have already started to give answers to these questions. In particular, there is an important difference in modern soliton physics, as compared with that in earlier decades. Due to outstanding technical developments in quantum optics $[9,10]$ we are now able to generate and observe solitons under conditions where operational measurements of both classical and quantum effects are routinely possible [11]. This means that soliton theory is not just a branch of applied mathematics, but is a true physical science in which theoretical predictions often lead immediately to an experimental verification in the laboratory. 
Accordingly, we focus here on the novel and unexpected features that have been found in both classical and quantum parametric field theories, which lead to higher-dimensional solutions that appear to be experimentally accessible. As well as reviewing some earlier work on degenerate parametric field theory, we give new results that are applicable to the nondegenerate case, and are particularly relevant to BoseEinstein condensates (BECs) or atom lasers $[12,13]$.

Before moving to the parametric case, we briefly remind the reader of the salient features of optical pulse propagation in single-mode fibres. The classical equations, which are approximately valid at large photon number, are given by:

$$
\mathrm{i} \frac{\partial \Phi}{\partial t}=-\frac{\hbar}{2 m} \frac{\partial^{2} \Phi}{\partial x^{2}}+\chi \Phi^{\dagger} \Phi^{2} .
$$

This is the NLS equation, where $\Phi=\langle\hat{\Phi}\rangle$ is the mean photon field amplitude, $m=\hbar / \omega^{\prime \prime}$ is the effective mass ( $\omega^{\prime \prime}$ is the dispersion coefficient; $m>0$ for anomalous dispersion), and $\chi$ is the coupling constant proportional to the third-order nonlinearity $\chi^{(3)}$. The soliton that occurs for $m / \chi<0$ is observed to propagate with solution $|\Phi| \propto \operatorname{sech}\left(\alpha x_{v}\right)$, where $x=x_{L}-v t, x_{L}$ is the laboratory frame coordinate and $v$ is the group velocity.

In quantum theory, there exists a set of bound states of this quantum field, which has a Hamiltonian [14] (in a frame moving at the group velocity) given by:

$$
\hat{H}=\frac{1}{2} \int \mathrm{d} x\left[\hbar^{2}\left|\partial \hat{\Phi}^{\dagger} / \partial x_{v}\right|^{2} / m+\hbar \chi \hat{\Phi}^{\dagger 2} \hat{\Phi}^{2}\right] .
$$

The corresponding di-photon eigenstate-as well as bound states with any number of photons-was discovered by Lieb and Liniger [14]:

$$
\left|\varphi^{(2)}\right\rangle=\iint \mathrm{d} x_{1} \mathrm{~d} x_{2} \mathrm{e}^{-\lambda\left|x_{1}-x_{2}\right|} \hat{\Phi}^{\dagger}\left(x_{1}\right) \hat{\Phi}^{\dagger}\left(x_{2}\right)|0\rangle,
$$

where $\lambda$ is a constant determined by the coupling strength and effective mass. However, a quantitative investigation shows that this di-photon solution is far too weakly bound to be observable in fibres. To relate these solutions to physical experiments, it is necessary to take into account the fact that practical experiments typically involve $10^{9}$ photons or more. Under these conditions, the bound states exist, but are not easily distinguished from each other, and are subject to disruption by thermal phonons. Thus, the simplest experiments observe classical soliton behaviour, which can be regarded as a superposition of quantum solutions [15]. These classical solitons are used (for example) in experimental communications systems. Predicted quantum effects [7] require measurement of quantum interference between the bound states. This leads to the observation of quantum squeezing $[8,16]$, quantum non-demolition collisions [17], and more recently-photon anti-bunching $[18,19]$.

\section{Classical parametric system}

In this section we review classical parametric soliton theory [20]. These solitons have a number of unusual features which have made them the subject of much recent theoretical
[21-29] and experimental [30] interest. First, these nonlinear solitary waves involve a wave coupled to its second harmonic, and hence rely on the second-order ('parametric') or $\chi^{(2)}$ electromagnetic nonlinearity, rather than the $\chi^{(3)}$ nonlinearity of conventional optical fibre solitons. This means that the nonlinear phase-shift is proportional to $E$, rather than to $E^{2}$. This novel feature promises to allow an improved scaling behaviour, allowing solitons to form at lower intensities, than in the case of conventional $\chi^{(3)}$ solitons. Secondly, parametric solitons have a great deal of stability in higher dimensions, again unlike the conventional solitons of the NLS equations. Other optical solitary waves that are known to be stable in higher dimensions have the drawback of usually involving either very high intensities, or nonlinearities with slow response times, rather than the FS electronic response of typical parametric nonlinear media.

As against these advantages, we note that higherdimensional $(D+1)$ spatio-temporal solitons in parametric media [31-36] require positive (i.e. anomalous) dispersion at both carrier frequencies, as well as group-velocity matching between the frequencies. These requirements are difficult to satisfy with the usual nonlinear crystals, which are generally optimized to operate at optical, rather than infrared wavelengths. At these short wavelengths, most optical materials have normal dispersion, and therefore cannot form higher-dimensional spatio-temporal solitons. For this reason, so far experimental observations have been restricted to the spatial parametric solitons.

Recent theoretical developments have shown that these restrictions on bulk material properties can be greatly relaxed if structured material is used, in which the dispersion is enhanced using an embedded dielectric Bragg grating to create band-gaps near the frequencies of interest. Thus, it seems that spatio-temporal parametric solitons are feasible in higher-dimensional structured materials [37-40], even when prohibited by the bulk material dispersion properties. Solitons in enhanced dispersion devices of this type generally have greatly reduced interaction lengths, when compared with the corresponding solitons of the same duration, in bulk media. A possible application of these new solitons, to take advantage of these fast response times and short interaction lengths, is in all-optical logic gates or multiplexors.

Of more fundamental physical interest, is the realization that this type of interaction also occurs in nonlinear atom optics or BEC systems. Here the parametric coupling describes a coherent process of dimerization (diatomic molecule formation), that can lead, for example, to formation of a molecular BEC from an atomic condensate. BEC systems have potentially even stronger nonlinear interactions than in the photonic case. This allows a new class of nonlinear classical and quantum dynamics to be investigated, by taking advantage of recent developments in ultra-cold atomic trapping and cooling, leading to the observation of an atomic BEC [12].

\subsection{Parametric 'simultons'}

The equations for 'simulton' (simultaneous solitary waves) propagation in nondegenerate group-velocity matched planar 
$((2+1)$-dimensional) waveguides $[32,33,35,44]$ are:

$$
\begin{gathered}
\frac{\partial \phi_{j}}{\partial \xi}=\mathrm{i}\left[\frac{\partial^{2} \phi_{j}}{\partial \zeta^{2}}+\frac{\partial^{2} \phi_{j}}{\partial \tau^{2}}-\phi_{j}+\phi_{3-j}^{*} \phi_{3}\right], \quad(j=1,2), \\
\frac{\partial \phi_{3}}{\partial \xi}=\mathrm{i}\left[\frac{1}{\sigma} \frac{\partial^{2} \phi_{3}}{\partial \zeta^{2}}+\frac{1}{2} \frac{\partial^{2} \phi_{3}}{\partial \tau^{2}}-\gamma \phi_{3}+\phi_{1} \phi_{2}\right]
\end{gathered}
$$

where $\phi_{1}$ and $\phi_{2}$ describe scaled optical fields at frequencies $\omega_{1}$ and $\omega_{2} \simeq \omega_{1}$ respectively, and $\phi_{3}$ denotes the sum frequency field at $\omega_{3}=\omega_{1}+\omega_{2}$. The scaled fields have the property that $\left|\phi_{j}\right|^{2}$ is proportional to the photon number flux in the $j$ th mode. Typically, we are most interested in the case of degenerate frequencies $\left(\omega_{1}=\omega_{2}\right)$, and orthogonal polarizations, since this allows either input signal to be treated equivalently. Orthogonal polarizations can be rotated into each other with simple linear dielectric techniques. The term $\gamma$ is a phase mismatch term, between the signals and the second harmonic, and $\sigma$ describes the dispersion ratio between the second harmonic and signal fields. All the coordinates in these equations have been rescaled into a dimensionless form, as described in [35]; $\tau=(t-x / v) / t_{0}$ indicates the elapsed time, $\zeta=y / y_{0}-$ the transverse direction, while $\xi=x / x_{0}$ is the scaled $x$ coordinate. Note that the third-order nonlinearity has been neglected in the above equations.

The most well-studied case of spatio-temporal parametric solitons is that of the 'radially symmetric' solutions, in which $\sigma=2$, so that the ratio of dispersion matches the ratio of diffraction. This allows for solutions that have a radial symmetry in the pseudo-radial coordinate $r=\sqrt{\tau^{2}+\zeta^{2}}$. Substituting the ansatz $\phi_{j}=\phi_{j}(r)$ into the above equations and assuming $\phi_{1}=\phi_{2}$ gives:

$$
\begin{gathered}
\mathrm{i} \frac{\partial \phi_{1}}{\partial \xi}+\frac{\partial^{2} \phi_{1}}{\partial r^{2}}+\frac{1}{r} \frac{\partial \phi_{1}}{\partial r}-\phi_{1}+\phi_{1}^{*} \phi_{3}=0, \\
\mathrm{i} \frac{\partial \phi_{3}}{\partial \xi}+\frac{1}{2}\left[\frac{\partial^{2} \phi_{3}}{\partial r^{2}}+\frac{1}{r} \frac{\partial \phi_{3}}{\partial r}\right]-\gamma \phi_{3}+\phi_{1}^{2}=0 .
\end{gathered}
$$

Therefore one can easily prove that there exist a family of simulton solutions for arbitrary value of $\gamma$ by following the known topological arguments [35] which interpret the coupled equations as a Newtonian system describing the motion of a virtual particle in two space dimensions. A non-topological (bright) simulton solution is represented as a closed path that passes through the point $(0,0)$. In one dimension, the path becomes a straight line when $\gamma=1$. Compared with one-dimensional spatial simultons, these two-dimensional simultons usually have higher amplitudes in order to compensate for the energy loss of the virtual particle due to the presence of 'friction'. The path also becomes a straight line at $\gamma=1$ in higher dimensions.

In the two-dimensional case, soliton solutions were found recently $[33,44,45]$ and were observed by Torruellas et al [30] experimentally in a configuration involving purely spatial solitons in bulk media, rather than the present case of spatio-temporal solitons. Three dimensional simultons were also discovered both approximately [32] and numerically [35]. In addition, these two- and three-dimensional simultons can be approximated by a Gaussian ansatz using a variational method [36], as we discuss later in more detail.
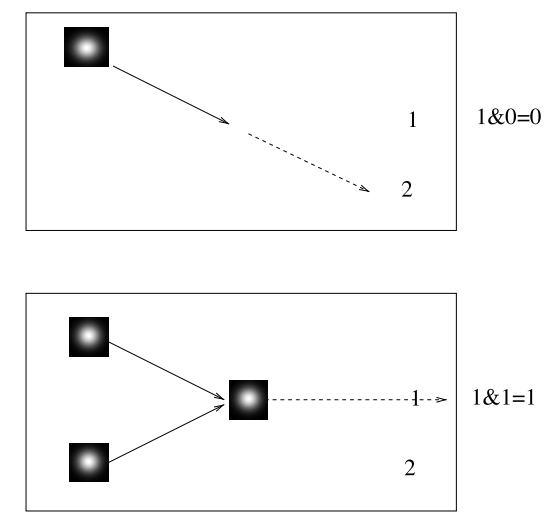

Figure 1. Y-geometry of colliding logic signals in a planar waveguide, showing inputs and output.

\section{All-optical parametric logic gate}

In order to illustrate a possible application of this type of soliton, we consider an AND-gate operating via non-collinear optical pulse collisions at the boundary of a nonlinear planar waveguide. This can be thought of as a Y-geometry, in which two pulses are input at an angle to each other, with the output logic pulse appearing at the bisector of the two input angles. This type of operation has several potential advantages over other proposed soliton or parametric logic gates [46-48]. Most importantly, the use of a Y-geometry means that the input lines to the logic gate can be easily and efficiently excited using planar waveguiding techniques. This is shown in figure 1. In addition, the use of temporal solitons has the advantage relative to purely spatial solitons, that short digital pulses are the natural signalling elements of the device. The combination of nonlinearity and dispersion provides an automatic re-shaping of the output signal, that can be then regenerated and reused in subsequent logic steps. Ideally, the two signals would have an orthogonal polarization, so that the interaction is not phase sensitive.

Here, we demonstrate numerically that an all optical AND-gate can be implemented by using a (2+1)-dimensional spatio-temporal simulton. The system simulated is a planar waveguide in which light is confined along one space dimension. In figure 2, we launch a Gaussian pulse with a small initial transverse velocity if the waveguide is viewed from above. Due to material dispersion, the pulse width increases and the pulse amplitude decreases while propagating. From figure 1, if we have a detector at position 1 , no (or very little) signal would be detected. In figure 3, we launch two Gaussian pulses simultaneously, both with the same parameters as those of the pulse in the first numerical experiment, except that one of the pulses has an opposite transverse velocity. This time, a simulton is formed and stable propagation of the simulton is observed. If we have a detector at position 1 , a signal would be detected.

It is important to note that no second-harmonic field is input. This is generated by the pulse-pulse interactions inside the nonlinear waveguide, so that the essential signalling element is a pulse of just one frequency, as in most optical communications systems. While the inputs have different polarizations, the existence of all-optical techniques for efficient polarization rotation, means that the two input 


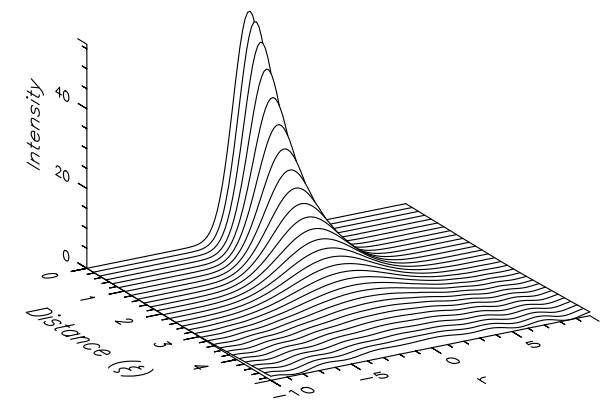

Figure 2. Propagation of a single input pulse, showing a strongly dispersed pulse being absorbed at the waveguide boundary. Only the cross section of $v_{1}$ is shown here. The initial pulses were: $v_{1}=7.5 \exp \left(-r^{2} / 4\right), v_{2}=0, v_{3}=0$, with $\gamma=1$ and $\sigma=2$.

(a)

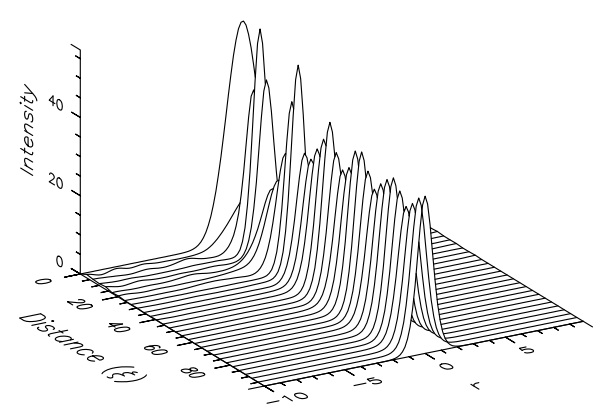

(b)

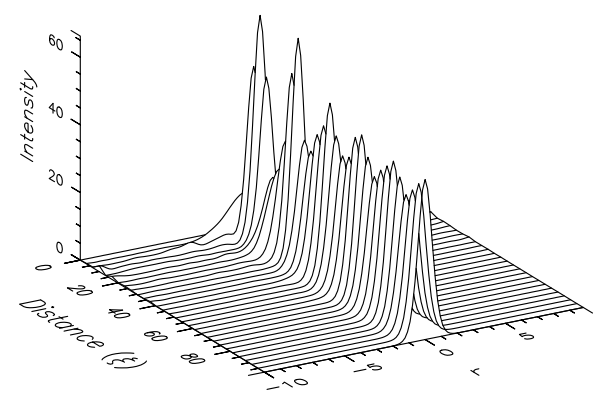

Figure 3. Propagation of two input pulses, forming a stable coupled solitary wave which is neither dispersed nor absorbed, resulting in an AND operation. (a) Cross section of $v_{1} ;(b)$ cross section of $v_{3}$. The initial pulses were: $v_{1}=7.5 \exp \left(-r^{2} / 4\right)$, $v_{2}=7.5 \exp \left(-r^{2} / 4\right), v_{3}=0$. Both input pulses, $v_{1}$ and $v_{2}$, are moving towards each other with a relative speed of 0.2 and an initial distance of 0.2 between them at $\xi=0$. Note that there is no second harmonic, $v_{3}$, at $\xi=0$. As before, $\gamma=1$ and $\sigma=2$.

signals are effectively interchangeable. The output has very similar characteristics to the input (after the second-harmonic is discarded), and overall gain could be implemented via further interactions with a second-harmonic pump. Thus, the desirable properties of electronic gates can be implemented in this logic, except with greater speed.

In summary, we have demonstrated that an all optical AND-gate can be implemented using the collision of two pulses in a planar nonlinear parametric waveguide. The switching mechanism is not phase sensitive and therefore has advantages over other phase sensitive switching proposals. It is not strongly limited by relaxation times, since the electronic nonlinearities are very fast, being of the order of fs. Due to the use of a $\chi^{(2)}$ rather than $\chi^{(3)}$ nonlinearity, we expect power requirements to be extremely low, with pulse energy requirements in the $\mathrm{pJ}$ range. This desirable combination of low switching energies and fast response times is difficult to implement using $\chi^{(3)}$-type nonlinearities. The device size is determined by the dispersion characteristics of the waveguide, and can be reduced by using enhanced dispersion devices based on Bragg gratings [37-40].

\section{Quantum parametric system}

Quantum solitons [6] or bound states of interacting fields are generalizations of the nonlinear solitonic solutions of classical wave theory, to include quantum fields. Exactly soluble cases include the many-body bound states of bosons interacting via $\delta$-function interactions in one space dimension. This model (often called the NLS model) was solved by Lieb et al [14]. Recently it was predicted that this soluble model could lead to experimentally observable quantum effects including quantum squeezing in optical fibre solitons $[7,15]$. This prediction has now been verified experimentally [8].

Exactly soluble models are generally restricted to one space dimension-except for Laughlin's highly innovative theory of two-dimensional electron gas in an external magnetic field [49], which was able to explain the fractional quantum Hall effect [50]. Similar techniques have been recently proposed for treating interacting Bose gases in higher dimensions [51], in the limit of very weak couplings. Other exact solutions in two or three dimensions are usually for physically inaccessible models, like the quantum DaveyStewartson model [52].

In a recent paper [53], we showed that it is possible to obtain an exact solution in one, two and three space dimensions, in a nonlinear quantum field theory that includes the most fundamental property that distinguishes quantum mechanics from quantum field theory - that is, the ability to create and destroy particles. The simplest cubic interaction involving two boson fields-the parametric interaction of the form $\Psi^{\dagger} \Phi^{2}$ - was analysed for bound states in higher dimensions, resulting in soluble cases with unusual and unexpected properties. This degenerate parametric theorywith similarities to the Friedberg-Lee [42] model of high- $\mathrm{T}_{C}$ superconductivity - has bound states in one space dimension [54], but is unstable (like the NLS model with an attractive $\delta$-function potential) in higher dimensions. However, unlike the NLS model, the instability does not occur at the classical level [31]. With the inclusion of an additional (repulsive) quartic interaction term in the Hamiltonian, a rigorous lower bound to the energy was proved to exist, and we demonstrated the existence of exact two-particle bound states in higher dimensions. These new types of quantum solitons have a finite binding energy, but the corresponding two-particle wavefunction has a zero radius; the point-like structure of these bound states can be termed a 'quantum singularity'. With a momentum cut-off imposed on the couplings, the bound states develop a finite radius.

In this paper, we extend these earlier results to include the non-degenerate case of parametric interaction of three 
distinct fields with either Bose or Fermi statistics (rather than two bosonic fields). Once again, the results demonstrate the existence of exact two-particle eigenstates in higher dimensions, having a point-like structure in space, with a finite energy. These hitherto unexpected eigenstates of a very simple form of quantum field theory appear to us to provide a theoretical counter-example to the usual belief in quantum field theory, that composite structures can be necessarily identified by their extended spatial properties. We also discuss the case of interactions in hybrid atomic/molecular Bose-Einstein condensates, where a cut-off is necessarily present, and extended spatial structure can form.

Here we give a summary of results in three space dimensions. A complete derivation in more general cases will be given in [55].

\subsection{Hamiltonian and di-photon eigenstate}

The quantum effective Hamiltonian $\left(\hat{H}=\hat{H}_{0}+\hat{H}_{\text {int }}\right)$ we consider has the following simplified form $[7,41]$ :

$$
\begin{gathered}
\hat{H}_{0}=\hbar \int \mathrm{d}^{3} \boldsymbol{x}\left[\sum_{i=1}^{3} \frac{\hbar}{2 m_{i}}\left|\nabla \hat{\Phi}_{i}\right|^{2}+\Delta \omega \hat{\Phi}_{3}^{\dagger} \hat{\Phi}_{3}\right], \\
\hat{H}_{i n t}=\hbar \int \mathrm{d}^{3} \boldsymbol{x}\left[\chi\left(\hat{\Phi}_{1} \hat{\Phi}_{2} \hat{\Phi}_{3}^{\dagger}+\hat{\Phi}_{1}^{\dagger} \hat{\Phi}_{2}^{\dagger} \hat{\Phi}_{3}\right)+\kappa \hat{\Phi}_{1}^{\dagger} \hat{\Phi}_{1} \hat{\Phi}_{2}^{\dagger} \hat{\Phi}_{2}\right] .
\end{gathered}
$$

Here $\hat{\Phi}_{1,2}$ and $\hat{\Phi}_{3}$ represent three quantum fields of masses $m_{i}(i=1,2,3)$. Their commutation relations in the case of Bose fields are given by $\left[\hat{\Phi}_{i}(\boldsymbol{x}), \hat{\Phi}_{j}^{\dagger}\left(\boldsymbol{x}^{\prime}\right)\right]=\delta_{i j} \delta\left(\boldsymbol{x}-\boldsymbol{x}^{\prime}\right)$. Although we have specified Bose statistics for definiteness, we note here that some of our main results for quantum solitons are also valid if the fields $\hat{\Phi}_{1}$ and $\hat{\Phi}_{2}$ obey Fermi statistics and anticommutation relations, as in the 's-channel' model of Friedberg-Lee [42]. Similarly, the case where $\hat{\Phi}_{2}$ and $\hat{\Phi}_{3}$ are fermionic (as in the Lee-Van Hove model of nuclear interactions [43]) is also tractable. In addition, $\hbar \Delta \omega$ is the formation energy of the field $\hat{\Phi}_{3}$, while $\chi$ and $\kappa$ are the coupling constants responsible for the parametric and quartic couplings, respectively. The role of the higher-order (quartic) interaction term that we have included in the quantum theory will be discussed below.

An example of a physical system that can directly be treated by the Hamiltonian (6) is a coupled atomic/molecular BEC, where the parametric coupling represents a coherent process of formation of di-atomic molecules ( $\hat{\Phi}_{3}$-field $)$ from pairs of atoms ( $\hat{\Phi}_{1}$ and $\hat{\Phi}_{2}$ fields) either of distinct atomic species, or in distinct quantum states. The quartic term represents atomic S-wave scattering [56], such that

$$
\kappa=\frac{2 \pi \hbar a}{m},
$$

where $a$ is the $\mathrm{S}$-wave scattering length between two atoms from different species and $m=m_{1} m_{2} /\left(m_{1}+m_{2}\right)$ is the corresponding reduced mass. In the case of identical $\hat{\Phi}_{1}$ and $\hat{\Phi}_{2}$ fields (i.e. degenerate interaction) this matter-wave analogue of the nonlinear optical process of frequency doubling has been considered in $[57,58]$, while the results of the present nondegenerate case are discussed in section 5 .

Employing the above Hamiltonian with photonic interactions (in which case the couplings $\chi$ and $\kappa$ are proportional to the Bloembergen quadratic and cubic nonlinearities, $\chi^{(2)}$ and $\chi^{(3)}$, and $\Delta \omega$ is a phase mismatch term) would first require that the longitudinal coordinate, say $x$, is defined in a moving frame with $x=x_{L}-v t$, where $x_{L}$ is the laboratory frame and $v=\partial \omega_{i} / \partial k$ is the group velocity which is assumed equal at all three carrier frequencies $\omega_{i}$ of the fields. In addition, the effective masses will be, in general, different in the longitudinal and transverse directions, $m_{i \|} \neq$ $m_{i \perp}$; the effective longitudinal mass $m_{i \|}=\hbar / \omega_{i}^{\prime \prime}$ is due to the group-velocity dispersion, where $\omega_{i}^{\prime \prime}=\partial^{2} \omega_{i} / \partial k^{2}$ is the dispersion coefficient in the $i$ th frequency band, while the transverse mass $m_{i \perp}=\hbar \omega_{i} / v^{2}$ is due to diffraction. This means that the kinetic energy terms in the above Hamiltonian must be modified, to incorporate this asymmetry, according to: $\left(1 / m_{i}\right) \nabla^{2} \rightarrow\left(1 / m_{i \|}\right) \partial_{x}^{2}+\left(1 / m_{i \perp}\right) \nabla_{\perp}^{2}$. In this paper we only consider the symmetric case $m_{i \|}=m_{i \perp} \equiv m_{i}$; the general asymmetric case and required modifications (similar to those in [53]) of the corresponding results will be treated elsewhere [55].

To construct the general candidate for the eigenstate to the Hamiltonian (6) we note that the parametric interaction here conserves a generalized particle number (Manley-Rowe invariant) equal to: $\hat{N}=\int \mathrm{d}^{3} \boldsymbol{x}\left[\left|\hat{\Phi}_{1}\right|^{2}+\left|\hat{\Phi}_{2}\right|^{2}+2\left|\hat{\Phi}_{3}\right|^{2}\right]$. We therefore search for states that are eigenstates of $\hat{H}$ and $\hat{N}$. In the case of two-particle bound state $(N=2)$, that we study in this paper, this must be a superposition state of the form:

$$
\begin{aligned}
\left|\varphi^{(2)}\right\rangle & =\left[\int \mathrm{d}^{3} \boldsymbol{x} P(\boldsymbol{x}) \hat{\Phi}_{3}^{\dagger}(\boldsymbol{x})\right. \\
& \left.+\iint \mathrm{d}^{3} \boldsymbol{x} \mathrm{d}^{3} \boldsymbol{y} Q(\boldsymbol{x}, \boldsymbol{y}) \hat{\Phi}_{1}^{\dagger}(\boldsymbol{x}) \hat{\Phi}_{2}^{\dagger}(\boldsymbol{y})\right]|0\rangle,
\end{aligned}
$$

where $P$ and $Q$ are one- and two-particle wavefunctions, respectively.

We note that the interaction part of the Hamiltonian (6) could also contain other quartic interaction terms, such as self-interaction of the $\hat{\Phi}_{i}$ fields and cross-interaction between the $\hat{\Phi}_{1(2)}$ and $\hat{\Phi}_{3}$ fields. These have no effect on the two-particle eigenstates studied here, so that we only consider the quartic cross-interaction between the fields $\hat{\Phi}_{1}$ and $\hat{\Phi}_{2}$. Operating on equation (8) with the Hamiltonian (6) one can obtain that the eigenvalue problem $\hat{H}\left|\varphi^{(2)}\right\rangle=$ $E^{(2)}\left|\varphi^{(2)}\right\rangle$ is equivalent to a set of differential equations. To solve these equations we introduce the relative and centre-of-mass coordinates according to: $\boldsymbol{r}=\boldsymbol{x}-\boldsymbol{y}$, $\boldsymbol{R}=\left(m_{1} \boldsymbol{x}+m_{2} \boldsymbol{y}\right) /\left(m_{1}+m_{2}\right)$. Taking into account translational invariance, we may then assume that $P(x)=$ $P_{0} \exp (\mathrm{i} \boldsymbol{K} \cdot \boldsymbol{x})$, where $\boldsymbol{K}$ is the soliton momentum. As a consequence, $Q(\boldsymbol{x}, \boldsymbol{x})$ will then be proportional to $P(\boldsymbol{x})$. Hence, we may look for the general expression of $Q(\boldsymbol{x}, \boldsymbol{y})=$ $g(\boldsymbol{r}) P(\boldsymbol{R})$. Dividing the energy into centre-of-mass and relative components $E^{(2)}=E_{c}+E_{r}$, we then solve the equation for $P(\boldsymbol{R})$, yielding $P(\boldsymbol{R})=P_{0} \exp (\mathrm{i} \boldsymbol{K} \cdot \boldsymbol{R})$, with $K^{2}=2\left(m_{1}+m_{2}\right) E_{c} / \hbar^{2}$. As a result, we obtain:

$$
E^{(2)}=\hbar^{2} K^{2} /\left(2 m_{3}\right)+\hbar \Delta \omega+\hbar \chi g(0) .
$$

The remaining equation, for the $g(r)$-function, is rewritten as

$$
\frac{1}{2 m} \nabla^{2} g(\boldsymbol{r})-\mu^{2} g(\boldsymbol{r})=\frac{1}{\hbar}[\chi+\kappa g(0)] \delta(\boldsymbol{r}),
$$


where we have introduced a reduced mass $m=m_{1} m_{2} /\left(m_{1}+\right.$ $m_{2}$ ), which we note differs from the conventional definition by a factor of two. In addition, we have defined $\mu^{2}=$ $-E_{r} / \hbar^{2}=K^{2} /\left[2\left(m_{1}+m_{2}\right)\right]-E^{(2)} / \hbar^{2}$, implying that the following simultaneous equation must be satisfied:

$$
E^{(2)}=\frac{\hbar^{2} K^{2}}{2 m_{3}}+\hbar \Delta \omega+\hbar \chi g(0)=\frac{\hbar^{2} K^{2}}{2\left(m_{1}+m_{2}\right)}-\hbar^{2} \mu^{2} .
$$

Here $\mu$ must be real and positive for a localized bound state solution, with $\sqrt{2 m} \mu$ representing an inverse scale length, and $\hbar^{2} \mu^{2}$ can be interpreted as the binding energy of the soliton with the momentum $\boldsymbol{K}$.

Equations (10), (11) can easily be analysed using the Fourier transform method. In this approach we seek for a solution to equation (10) in the form $g(r)=$ $\int \mathrm{d}^{3} \boldsymbol{k} G(\boldsymbol{k}) \exp (\mathrm{i} \boldsymbol{k} \cdot \boldsymbol{r}) /(2 \pi)^{3}$, where $r=|\boldsymbol{r}|$. Expanding the $\delta$-function into a Fourier integral, we then obtain the Fourier transform equivalent to equation (10), solving which we find:

$$
g(r)=-\frac{q}{(2 \pi)^{3}} \int \mathrm{d}^{3} \boldsymbol{k} \frac{\exp (\mathrm{i} \boldsymbol{k} \cdot \boldsymbol{r})}{\mu^{2}+k^{2} /(2 m)},
$$

where $q \equiv[\chi+\kappa g(0)] / \hbar$

Using the definition of $q$, we then solve for $g(0)$ and obtain $g(0)=-\chi[\kappa+\hbar / f(\mu)]^{-1}$, where

$$
f(\mu)=\frac{1}{2 \pi^{2}} \int_{0}^{\infty} \mathrm{d} k \frac{k^{2}}{\mu^{2}+k^{2} /(2 m)} .
$$

This integral diverges; a strict treatment of this divergence, as a mathematical limit, is given in the next section, where it is attributed to $k_{\max } \rightarrow \infty$, with $k_{\max }$ being the upper limit in the integral. Hence we find that $g(0)=-\chi / \kappa$, and the energy eigenvalue $E^{(2)}$ is given by:

$$
E^{(2)}=\frac{\hbar^{2} K^{2}}{2 m_{3}}+\hbar \Delta \omega-\frac{\hbar \chi^{2}}{\kappa}=\frac{\hbar^{2} K^{2}}{2\left(m_{1}+m_{2}\right)}-\hbar^{2} \mu^{2} .
$$

With respect to $\mu$, this equation always has one positive solution if the following conditions are met: $\kappa>0$ and $\Delta \kappa-\hbar \chi^{2}<0$, where $\Delta \equiv \hbar \Delta \omega+\hbar^{2} K^{2} /\left(2 m_{3}\right)-$ $\hbar^{2} K^{2} /\left[2\left(m_{1}+m_{2}\right)\right]$.

With the above result for $g(0)$ it also follows that $q=0$, and hence (see equation (12)) $g(r)=0$ if $r \neq 0$. That is, the exact bound state solution in three dimensions has a pointlike (zero-radius) structure, which is in the relative position of the $\hat{\Phi}_{1}$ and $\hat{\Phi}_{2}$ quanta.

\subsection{Cut-off dependent results}

The zero-radius behaviour of the two-particle quantum solitons in three space dimensions represents a rather unusual situation, since the classical counterpart of the bosonic theory has well-behaved, stable multidimensional nonlinear-optical soliton solutions [31]. This leads to the 'paradox' of how such a quantum field theory can describe real physical processes. To resolve this paradox, we note that physical applications usually involve some type of momentum cut-off.

In the case of nonlinear atom optics interactions, that we focus on here, the minimum relevant length scale for the cut-off $k_{\max }$ is the $\mathrm{S}$-wave scattering length $a$ (so that $k_{\max } \lesssim 2 \pi / a$ ) [56], and a cut-off also occurs in cases where fermionic fields are involved [42]. The eigenvalue problem $\hat{H}\left|\varphi^{(2)}\right\rangle=E^{(2)}\left|\varphi^{(2)}\right\rangle$ can now be analysed directly, in Fourier space, by introducing a cut-off dependent Fourier transform of $g(\boldsymbol{r})=\int_{|\boldsymbol{k}|=0}^{k_{\max }} \mathrm{d}^{3} \boldsymbol{k} G(\boldsymbol{k}) \exp (\mathrm{i} \boldsymbol{k} \cdot \boldsymbol{r}) /(2 \pi)^{3}$, so that the twoparticle eigenstate in Fourier space is written as:

$$
\begin{array}{r}
\left|\varphi^{(2)}(\boldsymbol{K})\right\rangle=\left[a_{3}^{\dagger}(\boldsymbol{K})+(2 \pi)^{-3 / 2} \int_{|\boldsymbol{k}|=0}^{k_{\max }} \mathrm{d}^{3} \boldsymbol{k} G(\boldsymbol{k})\right. \\
\left.\quad \times a_{1}^{\dagger}\left(\frac{m_{1} \boldsymbol{K}}{m_{1}+m_{2}}+\boldsymbol{k}\right) a_{2}^{\dagger}\left(\frac{m_{2} \boldsymbol{K}}{m_{1}+m_{2}}-\boldsymbol{k}\right)\right]|0\rangle,
\end{array}
$$

where $a_{i}(\boldsymbol{k})$ is the Fourier component of $\hat{\Phi}_{i}$, and the cut-off is in the relative momentum of the fields $\hat{\Phi}_{1}$ and $\hat{\Phi}_{2}$.

The simultaneous equations for the eigenstate still have the form of the Fourier transform of equations (10) and (11), except that they are now valid for $|\boldsymbol{k}|<k_{\max }$. In order to evaluate the binding energy and the effective radius, we next solve these equations for $g(0)$, and obtain $g(0)=$ $-\chi\left[\kappa+\hbar / f\left(\mu, k_{\max }\right)\right]^{-1}$. Here the cut-off structure function is given by:

$$
\begin{gathered}
f\left(\mu, k_{\max }\right)=\frac{1}{(2 \pi)^{3}} \int_{|k|=0}^{k_{\max }} \frac{\mathrm{d}^{3} \boldsymbol{k}}{\mu^{2}+(k)^{2} /(2 m)} \\
=\frac{m}{\pi^{2}}\left[k_{\max }-\mu \sqrt{m} \tan ^{-1}\left(\frac{k_{\max }}{\mu \sqrt{2 m}}\right)\right],
\end{gathered}
$$

and thus has a linear divergence as $k_{\max } \rightarrow \infty$. The effect of this divergence depends on whether or not the additional quartic interaction is present. If it is present (with $\kappa>0$ ), there are exact solutions without cut-off (i.e. $k_{\max } \rightarrow \infty$ ), and $g(0)=-\chi / \kappa$, so that the energy eigenvalue $E^{(2)}$ takes the form of equation (14), and $g(r)=0$ if $|r|>0$. In other words, the solution has a finite energy (unlike the energy divergence in the NLS model with an attractive $\delta$-function potential) but zero radius in the limit of $k_{\max } \rightarrow \infty$. If, however, $\kappa \leqslant 0$, we must impose a finite cut-off on the couplings to prevent an energy divergence. Simultaneously, a finite cut-off prevents singularities in space.

With a finite cut-off, the general result for the energy eigenvalue $E^{(2)}$ is given by:

$$
\begin{aligned}
E^{(2)}\left(k_{\max }\right) & =\frac{\hbar^{2} K^{2}}{2 m_{3}}+\hbar \Delta \omega-\hbar \chi^{2}\left[\kappa+\frac{\hbar}{f\left(\mu, k_{\max }\right)}\right]^{-1} \\
& =\frac{\hbar^{2} K^{2}}{2\left(m_{1}+m_{2}\right)}-\hbar^{2} \mu^{2}
\end{aligned}
$$

where $\mu$ must be positive for a localized bound state. Analysis of this equation shows that a positive solution for $\mu$ is always available, if $\kappa>0$ and $\Delta\left[\kappa+\pi^{2} \hbar /\left(m k_{\max }\right)\right]$ $-\hbar \chi^{2}<0$.

In the simplest case of $\kappa=0$ and $\Delta=0$, and in the limit $k_{\max } \gg \mu \sqrt{2 m}$ one can write down simple approximate results for the binding energy:

$$
E^{b}=\hbar^{2} \mu^{2} \simeq \frac{\chi^{2} m k_{\max }}{\pi^{2}} .
$$

The effective radius of the quantum soliton is defined as $R=1 /(\mu \sqrt{2 m})$, since this determines the characteristic distance over which the two-particle wavefunction can decay. 


\section{BEC interactions}

An example of a physical system that can directly be treated by the Hamiltonian (6) is a coupled atomic/molecular BEC, where $\hat{\Phi}_{1,2}$ represent two atomic fields, $\hat{\Phi}_{3}$ is the molecular field, $m_{1,2}$ and $m_{3}$ are the atomic and molecular masses $\left(m_{3}=m_{1}+m_{2}\right)$, the coupling constant $\chi$ relates to the molecular formation (coherent dimerization) rate, $\kappa$ is the effective cross-scattering between the atoms of two different species, and $\hbar \Delta \omega$ is the bare formation energy of the molecular species. We note that the Hamiltonian should also contain quartic terms due to scatterings of atoms within each species, as well as molecule-molecule and atom-molecule scattering terms. These, however, do not affect the two-particle quantum soliton solutions given earlier. In the many-particle regime, that we treat below variationally, we neglect the contribution of these quartic couplings as compared to the $\kappa$-coupling or assume a low particle density limit. Physical mechanisms that can realize coherent atomic dimerization and produce ultracold molecules include Feshbach resonance and Raman photoassociation $[59,60]$. Feshbach resonances have already been observed [61], while experiments of this type with the Raman photoassociation are underway [62] in the case of homogeneous (degenerate) BEC the theory of which is given elsewhere [57, 58,63].

In such coupled atomic/molecular BEC systems the twoparticle (di-boson) quantum soliton solutions are 'dressed' molecules each of which exists in a superposition with a pair of atoms. With a characteristic $\chi$-value estimate of about $\chi \sim 10^{-6} \mathrm{~m}^{3 / 2} \mathrm{~s}^{-1}[60,62,63]$, the atomic masses $m_{1} \simeq m_{2} \sim 10^{-25} \mathrm{~kg}$, and the longest S-wave scattering length $a \sim 5 \mathrm{~nm}$, equation (14) results in the quantum soliton binding energy of: $E^{b}=\hbar \chi^{2} / \kappa \simeq 10^{-11} \mathrm{eV}$, for $\Delta \omega=0$. If we include both effects of the quartic repulsive term $\kappa=2 \pi \hbar a / m$ and the momentum cutoff, and assume that the scattering length $a$ provides a natural cutoff at $k_{\max } \sim 2 \pi / a$, then the binding energy from equation (17) is reduced (for $\Delta \omega=0$ ) to $E^{b} \simeq 4 \hbar \chi^{2} /(5 \kappa)$, which is very close to the idealized result from equation (14) and is comparable to achievable temperatures in current BEC experiments.

Of more importance, from the point of view of BEC experiments, are $N$-particle eigenstates and the ground state energy of this quantum many-body system. While this is a difficult problem, some important results can be obtained using a variational approach. Here we consider two important examples: (i) a variational ansatz that corresponds to $N / 2$ (where we assume $N$ is even) independent di-bosons [58], and (ii) a coherent or mean-field theory (MFT) ansatz. A remarkable result that emerges with the treatment of the first type of ansatz is that, in the limit $k_{\max } \rightarrow \infty$, it turns into the exact eigenstate and provides the exact ground state energy of: $E^{(N)}=(N / 2) E^{(2)}=\hbar(N / 2)\left[\Delta \omega-\hbar \chi^{2} / \kappa\right]$. The ground state energy has no lower bound as $\kappa \rightarrow 0$. This is in contrast to the mean-field behaviour corresponding to the classical Hamiltonian energy. The classical Hamiltonian is known to have rigorous lower bound and to support classical solitons [31].

With a finite cut-off, the ansatz corresponding to $N / 2$ independent di-bosons, is no longer the exact eigenstate, and therefore does not necessarily result in the lowest possible energy. The corresponding variational estimate of the energy, in three dimensions and in the low density limit, is (for $\Delta \omega=0)$ :

$$
\tilde{E}^{(N)}=(N / 2) E^{(2)}\left(k_{\max }\right)-2 N \hbar \chi^{2} /(5 \kappa) .
$$

The second type of ansatz that we employ herethe coherent or mean-field theory ansatz $\left|\varphi_{C}^{(N)}\right\rangle=$ $\exp \left[\int \mathrm{d}^{3} \boldsymbol{x} \sum_{i=1}^{3} \Phi_{i}(\boldsymbol{x}) \hat{\Phi}_{i}^{\dagger}(\boldsymbol{x})\right]|0\rangle$-may provide a lower energy at large $N$. To show this, we use trial functions $\Phi_{i}(\boldsymbol{x})$ in the form of Gaussians, and assume that $\Phi_{1}(\boldsymbol{x})=\Phi_{2}(\boldsymbol{x})$. The final result for the corresponding minimum energy is considerably simplified in the region where the parametric coupling is dominant, so that one can neglect the terms due to $\Delta \omega$ and the inter-atomic scattering $k$ (as well as the intraatomic, intra-molecular and atom-molecule scatterings). In this region, and for $m_{3}=m_{1}+m_{2}$ and $m_{1}=m_{2}$ (so that $m_{3}=4 m$ ), we obtain a coupled atomic/molecular Bose condensate minimum energy of:

$$
\tilde{E}_{C}^{(N)}=-C N^{3} m^{3}(\chi)^{4} / \hbar^{2},
$$

where $C$ is a constant given by $C \simeq 1.2 \times 10^{-5}$. The relevant length scales, corresponding to the soliton widths, are nearly identical for the three fields and are given by: $l \simeq 1.7 \times$ $10^{2} \hbar^{2} N^{-1}(m \chi)^{-2}$. Comparing equations (19) and (20) we can see that the coherent or MFT ansatz corresponding to the classical theory becomes more favourable at a critical boson number $N>N_{\mathrm{cr}}=(m \chi / \hbar)^{-1}(5 \mathrm{Cm} \kappa / 2 \hbar)^{-1 / 2}$, as $\tilde{E}_{c}^{(N)}<$ $\tilde{E}^{(N)}$. With the parameter values for $\chi$ and the scattering length $a$ (and therefore $\kappa$ ) given earlier and characteristic for current BEC experiments, it turns out, however, that the role of the repulsive term $\kappa$ is not negligible in the mean-field theory analysis. In general, the effect of the repulsive quartic interaction is towards destabilizing the soliton formation. The result of minimization of the corresponding MFT energy is no longer given by a simple cubic dependence on $N$ as in equation (20). Instead, our numerical analysis shows that the variational results corresponding to MFT and di-boson ansatz become comparable to each other and close to a linear dependence on $N$. Further detailed analysis and comparison is required in this regime, with a more careful treatment of the origin of the momentum cutoff, as well as treatment of finite density effects and the role of other quartic couplings, such as molecule-molecule and atom-molecule scatterings. This, however, is beyond the scope of the present paper. We also note that the question of soliton formation and its stability under both the parametric and quartic couplings, even at the pure classical level and in a three-dimensional environment, has not been analysed yet.

Thus, at low particle density, the formation of individual 'dressed' molecules is favoured, as atoms couple to molecules in a particle-like way. These dressed states have interesting properties, reminiscent of Cooper pairs, but cannot be described by the classical parametric soliton equations. At large density (but not too large so that S-wave scattering is dominant) the coherent coupling of three entire condensates is dominant. With large enough parametric coupling, and provided other recombination 
processes are negligible, there are coherent nonlinear wavelike interactions between the atomic and the molecular Bose condensates (just as in nonlinear optics), which make it possible to form stable, three-dimensional, BEC solitons.

More than this, the coherent nonlinear dynamics of these BEC interactions may result in completely novel type of chemical reactions at ultralow temperatures. This 'superchemistry' behaviour can give enormous (Boseenhanced) chemical reaction rates $\left(\propto N_{1} \sqrt{N_{3}}\right)$, due to the effect of bosonic stimulated emission, similar to lasing. This is in sharp contrast to the predictions of chemical kinetics, where the rates do not depend on the number of product particles, and go to zero at low temperatures, according to the Arrhenius law.

\section{Summary}

In summary, we have reviewed some recent developments in soliton theory, focussing on nondegenerate parametric solitons (or simultons), in two and three dimensions. In the classical case of a photonic soliton, a possible application to a classical logic gate is given. In the quantum soliton case, we have given relevant results in three dimensions for a Bose condensate of two different atomic states, in which the interaction is due to coherent molecule formation. As in the degenerate parametric case, the di-boson quantum soliton without a cut-off has a quantum point-like (zeroradius) structure. The reason for this behaviour-which is quite different to the classical solutions-is the inherently nonclassical structure of the bound state, which is a quantum superposition state. We note that most previous analyses of quantum solitons treated cases where the quantum soliton was at least qualitatively similar to the corresponding classical theory. This is not the case here, at least at low densities.

The physical interpretation of these bound states is that they are a superposition of states containing either one or two particles. The basic solution can formally be written as $A\left|0_{1}, 0_{2}, 1_{3}\right\rangle+B\left|1_{1}, 1_{2}, 0_{3}\right\rangle$, which we note is related to states employed in quantum computation applications. Thus, one might envisage molecule formation as part of a logic gate in a quantum computer. With the inclusion of momentum cutoffs on the nonlinear couplings, the two-particle bound state has a finite radius even in the simplest case of pure parametric interaction-i.e. without the quartic term.

Most significantly, these quantum solitons form in physically testable regimes. Our estimates for characteristic sizes and soliton binding energies result in much more realistic values than examples of quantum solitons based on pure quartic couplings with cubic optical nonlinearities [64]. Another promising experimental environment is available with current BEC technology. It seems possible that one could investigate a transition between the di-boson regime, and the semi-classical 'superchemistry' regime of coupled condensates, which could dominate at large particle number, provided inter-particle repulsion is small. In this regime stable matter-wave solitons may form in three dimensions, implying an intense stable atom/molecular laser output. We note that earlier examples of matter wave BEC type solitons (see, e.g., [65] and references therein) were only for a onedimensional geometry.
Finally, the bosonic character of the fields is not relevant for the quantum bound state theory derived here. Exactly the same results would occur if fermionic fields were involved, and we changed the corresponding commutation relations to anticommutators. In this respect, the present theory differs from the previous degenerate case $[53,58]$, where the results were only applicable to bosonic fields. This suggests that part of these results (but not the semi-classical soliton theory) could be extended to possible atomic fermionic superconductors, in which coupling between fermionic atoms is enhanced by the coherent production of bosonic molecules. Thus, the applications of quantum soliton theory in experimentally accessible regimes is a rich and rapidly developing science, built on the novel technologies and ideas of quantum and atom optics.

\section{References}

[1] Scott-Russell J 1840 Proc. R. Soc. Edinburgh 14 47-102 Scott-Russell J 1844 Proc. R. Soc. Edinburgh $1433-4$

[2] Korteweg D J and de Vries G 1895 Phil. Mag. 39422

[3] Coleman S 1985 Aspects of Symmetry (Cambridge: Cambridge: University Press)

[4] Hasegawa A and Tappert F 1973 Appl. Phys. Lett. 23 142-4

[5] Mollenauer L F, Stolen R H and Gordon J P 1980 Phys. Rev. Lett. 45 1095-98

[6] Christ N H and Lee T D 1975 Phys. Rev. D 121606 Lee T D 1988 Particle Physics and Introduction to Field Theory (London: Harwood Academic)

[7] Carter S J et al 1987 Phys. Rev. Lett. 581841 Drummond P D and Carter S J 1987 J. Opt. Soc. Am. B 4 1565

[8] Rosenbluh M and Shelby R M 1991 Phys. Rev. Lett. 66153

[9] Walls D F 1983 Nature 306141

[10] Slusher R E et al 1987 Phys. Rev. Lett. 592566

[11] Drummond P D, Shelby R M, Friberg S R and Yamamoto Y 1993 Nature 365307

[12] Anderson M H et al 1995 Science 269198 Bradley C C et al 1995 Phys. Rev. Lett. 751687

Davis K B et al 1995 Phys. Rev. Lett. 753969

Burnett K 1996 Contemp. Phys. 371

Davis K B, Mewes M-O and Ketterle W 1995 Appl. Phys. B 60155

[13] Mewes M-O et al 1997 Phys. Rev. Lett. 78582

Wiseman H and Collet M J 1995 Phys. Lett. A 202246

Guzman A M, Moore M and Meystre P 1996 Phys. Rev. A 53 977

Holland M et al 1996 Phys. Rev. A 54 R1757

[14] Lieb E H and Liniger W 1963 Phys. Rev. 1301605

McGuire I B 1964 J. Math. Phys. 5622

Yang C N 1967 Phys. Rev. 1681920

Thacker H B 1981 Rev. Mod. Phys. 53253

[15] Lai Y and Haus H A 1989 Phys. Rev. A 40844 Haus H A and Lai Y 1990 J. Opt. Soc. Am. B 7386

[16] Bergman K and Haus H A 1991 Opt. Lett. 16663

[17] Friberg S R, Machida S and Yamamoto Y 1992 Phys. Rev. Lett. 693165

[18] Werner M 1996 Phys. Rev. A 54 R2567 Werner M J and Friberg S R 1997 Phys. Rev. Lett. 794143

[19] Spalter S, Burk M and Leuchs G 1997 Europhys. Lett. 38335

[20] Karamzin Y N and Sukhorukov A P 1975 Zh. Eksp. Teor. Fiz. 68 834-47

Karamzin Y N and Sukhorukov A P 1974 Moscow Univ. Phys. Bull. 20 339-42

[21] Werner M J and Drummond P D 1993 J. Opt. Soc. Am. B 10 2390-3

[22] Karpierz M A and Sypek M 1994 Opt. Commun. 110 75-9

[23] Menyuk C R, Schiek R and Torner L 1994 J. Opt. Soc. Am. B 11 2434-43 
[24] Hayata K and Koshiba M 1994 Phys. Rev. A 50 675-9

[25] Buryak A V and Kivshar Y S 1994 Opt. Lett. 191612

[26] Torner L, Menyuk C R and Stegeman G I 1995 J. Opt. Soc. Am. B 12 889-97

[27] Torner L 1995 Opt. Commun. 114 136-40

[28] Torner L, Menyuk C R and Stegeman G I 1994 Opt. Lett. 19 1615-17

[29] Buryak A V and Kivshar Y S 1995 Phys. Rev. A 51 R41-4

[30] Torruellas W E et al 1995 Phys. Rev. Lett. 74 5036-9

[31] Kanashov A A and Rubenchik A M 1981 Physica D 4122

[32] Hayata K and Koshiba M 1993 Phys. Rev. Lett. 71 3275-8

[33] Torner Let al 1995 Opt. Lett. 20 13-15

[34] Bergé L et al 1995 Phys. Rev. A 52 R28

[35] He H, Werner M and Drummond P D 1996 Phys. Rev. E 54 896

[36] Malomed B A et al 1997 Phys. Rev. E 56 4725-35

[37] Peschel T et al 1997 Phys. Rev. E 554730

[38] Conti C, Trilo S and Assanto G 1997 Phys. Rev. Lett. 782341

[39] He H and Drummond P D 1997 Phys. Rev. Lett. 784311

[40] He H and Drummond P D 1998 Phys. Rev. E 585025

[41] Caves C M and Crouch D D 1987 J. Opt. Soc. Am. B 41535 Raymer M G, Drummond P D and Carter S J 1991 Opt. Lett. 161189

Hillery M and Mlodinov L D 1984 Phys. Rev. A 301860 Drummond P D 1990 Phys. Rev. A 426845

[42] Friedberg R and Lee T D 1989 Phys. Rev. B 406745 Wang Y 1993 Phys. Lett. A 17619

[43] Lee T D 1954 Phys. Rev. 541329 Van Hove L 1955 Physica 21901 See also, Schweber S S 1961 An Introduction to Relativistic Quantum Field Theory (New York: Row Peterson)

[44] Buryak A V, Kivshar Y S and Steblina V V 1995 Phys. Rev. A 52 1670-4

[45] Steblina V V et al 1995 Opt. Commun. $118345-52$

[46] Drummond P D and Man W 1994 Opt. Commun. 105 99-103

[47] Assanto G 1995 Opt. Lett. 20 1595-7

[48] Asobe M et al 1997 Opt. Lett. 22 274-6

[49] Laughlin R B 1983 Phys. Rev. Lett. 501385

[50] Tsui D C, Störmer H L and Gossard H C 1982 Phys. Rev. Lett. 481559

[51] Wilkin N K, Gunn J M F and Smith R A 1998 Phys. Rev. Lett. 802265

[52] Schultz C L, Ablowitz M J and BarYaacov O 1987 Phys. Rev. Lett. 592825
Novel solitons in parametric amplifiers and atom lasers

Pang G D, Pu F-C and Zhao B-H 1990 Phys. Rev. Lett. 65 3227

Pang G D 1994 Phys. Lett. A 184163

[53] Kheruntsyan K V and Drummond P D 1998 Phys. Rev. A 58 2488

[54] Drummond P D and He H 1997 Phys. Rev. A 56 R1107

[55] Kheruntsyan K V and Drummond P D in preparation

[56] Fetter A L and Walecka J D 1991 Quantum Theory of Many-Particle Systems (New York: McGraw-Hill) Abrikosov A A, Gorkov L P and Dzyaloshinski I E 1963 Methods of Quantum Field Theory in Statistical Physics (New York: Dover)

Bogoliubov N N 1947 J. Phys. (USSR) 1123

Huang K 1963 Statistical Mechanics (New York: Wiley)

Proukakis N P, Burnett K and Stoof H T C 1998 Phys. Rev. A 571230

[57] Kheruntsyan K V and Drummond P D 1998 Phys. Rev. A 58 R2676

[58] Drummond P D, Kheruntsyan K V and He H 1998 Phys. Rev. Lett. 813055

[59] Vogels J M et al 1997 Phys. Rev. A 56 R 1067 Julienne P S et al 1998 Phys. Rev. A 58 R797

[60] Tommasini P, Timmermans E, Hussein M and Kerman A 1998 Preprint cond-mat/9804015

[61] Inouye S et al 1998 Nature 392151

Courteille Ph, Freeland R S and Heinzen D J 1998 Phys. Rev. Lett. 8169

[62] Heinzen D J Private communication

[63] Heinzen D J, Wynar R H, Drummond P D and Kheruntsyan K V submitted

[64] Deutsch I H and Chiao R Y 1992 Phys. Rev. Lett. 693627

Cheng Z and Kurizki G 1995 Phys. Rev. Lett. 753430

Deutsch I H, Chiao R Y and Garrison J C 1993 Phys. Rev. A 473330

Shnirman A G, Malomed B A and Ben-Jacob E 1994 Phys. Rev. A 503453

[65] Morgan S A, Ballagh R J and Burnett K 1997 Phys. Rev. A $\mathbf{5 5} 4338$

Reinhard W P and Clark C W 1997 J. Phys. B: At. Mol. Opt. Phys. 30 L785

Scott T F, Ballagh R J and Burnett K 1998 J. Phys. B: At. Mol. Opt. Phys. 31 L329

Zobay O, Pötting S, Meystre P and Wright E M 1999 Phys. Rev. A 59643 